\title{
Accretion Shocks in the Laboratory: Design of an Experiment to Study Star Formation
}

\author{
R. P. Younga,*, C. C. Kuranz ${ }^{\mathrm{a}}$, R. P. Drake ${ }^{\mathrm{a}}$, P. $\operatorname{Hartigan}^{\mathrm{b}}$ \\ ${ }^{a}$ University of Michigan, Department of Climate and Space Sciences and Engineering, 2455 \\ Hayward St., Ann Arbor, MI 48109 \\ ${ }^{b}$ Rice University, Physics and Astronomy Department, 6100 Main MS-61, Houston, Texas \\ 77005
}

\begin{abstract}
We present the design of a laboratory-astrophysics experiment to study magnetospheric accretion relevant to young, pre-main-sequence stars. Spectra of young stars show evidence of hotspots created when streams of accreting material impact the surface of the star and create shocks. The structures that form during this process are poorly understood, as the surfaces of young stars cannot be spatially resolved. Our experiment would create a scaled "accretion shock" at a major (several kJ) laser facility. The experiment drives a plasma jet (the "accretion stream") into a solid block (the "stellar surface"), in the presence of a parallel magnetic field analogous to the star's local field. We show that this experiment is well-scaled when the incoming jet has $\rho \sim 10^{-6}-10^{-5} \mathrm{~g} \mathrm{~cm}^{-3}$ and $u \sim 100-200 \mathrm{~km} \mathrm{~s}^{-1}$ in an imposed field of $B \sim 10 \mathrm{~T}$. Such an experiment would represent an average accretion stream onto a pre-main sequence star with $B \sim 700 \mathrm{G}$.
\end{abstract}

laboratory astrophysics; star formation; accretion; shocks; jets

\section{Introduction}

Accretion shocks form when material from an accretion disk impacts at the surface of the object growing at its center. The object's magnetic field

${ }^{*}$ Corresponding author

Preprint submitted to Journal of High Energy Density Physics

August 29, 2016

(C) 2017. This manuscript version is made available under the Elsevier user license http://www.elsevier.com/open-access/userlicense/1.0/ 
governs the interaction between the two through what it called magnetospheric accretion. Originally proposed by Königl [22], who extended the compact object work of Ghosh and Lamb [8, 9] to T Tauri stars (low-mass pre-main-sequence stars), the magnetospheric accretion model has material from the accretion disk lifted out of the plane of the disk and "funneled" along the star's magnetic field lines to its surface. Today, there is ample evidence that magnetospheric accretion occurs on T Tauri stars (see Bouvier et al. 3] and references therein). There is also evidence of magnetospheric accretion occurring on T Tauri stars' more massive counterpart, Herbig Ae/Be stars [29, 31, 12.

When the supersonic material impacts the surface of the young star- T Tauri or Herbig $\mathrm{Ae} / \mathrm{Be}$ - an accretion shock hot enough to emit soft X-rays ( $T \sim$ $\left.10^{6}-10^{7} \mathrm{~K}\right)$ forms. There is ample evidence of this in the X-ray spectra of T Tauri stars [21, 43, 42, 13, 2, 38, 4, 1], and evidence is growing that at least some Herbig Ae/Be stars exhibit X-ray-emitting accretion shocks as well [44, 45, 10, 6].

The mass accretion rate of a star $\left(\dot{M}\right.$, usually on the order of $10^{-8} \mathrm{M}_{\odot}$ per year) can be determined from the effect that these X-ray emitting accretion shocks have on the star's spectrum. The X-rays heat the surrounding photosphere, producing spots of hot plasma [5]. Compared to a similar non-accreting star, an accreting star ought to have excess emission in the optical and UV due to these spots, and its accretion rate can be calculated from the amount of excess emission [15, 46, 16, 30, 33].

However, these accretion rate calculations are only as good as the understanding of accretion shock structure behind them. Because the surfaces of young stars cannot be spatially resolved, the structure of accretion shocks has not been directly studied. For example, do accretion shocks penetrate the star's photosphere, potentially hiding much of the accretion shock's energy from observers? Or, do accretion shocks create large "splashes" when they hit the surface of the star, making it appear the shock covers more surface area than it actually does? Either of these scenarios would potentially change the calculated accretion rate significantly. 
Simulations of accretion shocks have largely served to underscore how complicated and inherently three-dimensional the systems are. Three-dimensional simulations by Romanova et al. 39. predict that accretion hotspots should be inhomogenous and irregularly shaped. Likewise, simulations by Orlando et al. [34] found that $\mathrm{T}$ Tauri accretion shocks can produce violent splash zones, particularly when the magnetic field strength is too low to the contain the accretion shock.

To this end, we propose our design of an experiment to produce a scaled laboratory version of an accretion shock. We would use a high-energy (several kJ) laser to make a supersonic plasma jet (the "accretion stream") and drive it into a solid block (the "stellar surface") in the presence of an imposed magnetic field. Section 2 establishes the connection between magnetic accretion on a pre-main-sequence star and the laboratory experiment and lays out the plasma parameters that we must seek to have a well-scaled experiment. Section 3 discusses design considerations of our experiment, and Section 4 presents our conclusions.

\section{Experimental Goals}

\subsection{Basic Configuration}

Laboratory astrophysics offers the ability to probe scaled astrophysical systems in an experimental setting [41, 37, 36. Examples of such experimental campaigns include the work of Kuranz et al. 25, who created Rayleigh-Taylor blast waves relevant to supernovae remnants; the work of Hartigan et al. [14, who created deflected supersonic jets relevant to Herbig-Haro objects; and the work of Krauland et al. 23, who created reverse shocks relevant to interacting binaries.

Our astrophysical system, an accreting young star, consists of (1) accreting material which collides with (2) the surface of the growing star in the presence of (3) a magnetic field parallel to the material's flow and perpendicular to the star's surface. Creating a scaled version of this system means translating these 
three into the lab, as seen in Figure 1 In the experiment, we drive a plasma jet (the "accreting material") into a solid block (the "stellar surface") with an imposed magnetic field.

\subsection{Dimensionless Numbers}

Astrophysical systems can never be scaled and reproduced perfectly in the lab. Having a worthwhile experiment, therefore, hinges on discerning which physical processes are most important and translating them into an experiment appropriately. Ryutov et al. [41] lays out a theoretical basis for doing so: one must ensure that dimensionless numbers (for example, Reynolds number) that define the system are at least in similar regimes.

There are five dimensionless numbers that concern the accretion shock experiment. The first two determine whether a shock can form in the first place, the second two determine the role of magnetic fields in the experiment, and the last is the Reynolds number, which relates the importance of viscosity in the system. The scaling criteria we impose are as follows:

1. $\mathcal{M}>1 \mathcal{M}$ is the Mach number, the ratio of the flow velocity of the jet to the speed of sound inside the jet; we need a supersonic jet in order to have a shock. Sound speed was calculated according to

$$
c_{s}=9.79 \times 10^{5} \sqrt{\frac{\gamma(Z+1) T_{e}}{A}} \mathrm{~cm} \mathrm{~s}^{-1},
$$

where $\gamma$ is the adiabatic index, $Z$ is the ionizations, $T_{e}$ is the temperature in $\mathrm{eV}$, and $A$ is the atomic mass in proton masses.

2. $\lambda_{\mathrm{MFP}}<\boldsymbol{L} \quad \lambda_{\mathrm{MFP}}$ the ion-ion mean free path inside the plasma and $L$ is the overall lengthscale of the experiment. We impose $\lambda_{M F P}<L$ in order to observe a shock in the experiment.

Mean free path was calculated according to

$$
\lambda_{\mathrm{MFP}}=\frac{1}{n_{i} \sigma_{90}^{i i} 4 \ln \Lambda_{i i}},
$$

where $n_{i}$ is the ion density, $\sigma_{90}^{i i}$ is the $90^{\circ}$ cross-section, and $\ln \Lambda_{i i}$ is the ion-ion Coulomb lambda. The $90^{\circ}$ cross-section was calculated according 
to

$$
\sigma_{90}^{i i}=\frac{\pi e^{4} Z^{4}}{m_{i} u^{4}},
$$

where $e$ is the charge of an electron, $Z$ is the average ionization, $m_{i}$ is the ion mass, and $u$ is the relevant velocity (in this case the flow velocity).

This criterion $\left(\lambda_{\mathrm{MFP}}<L\right)$ also ensures that our plasma is collisional.

Park et al. 35] give the condition for a collisionless plasma as $L \ll \lambda_{\mathrm{MFP}}$, where $\lambda_{\text {MFP }}$ is the ion-ion MFP of the heaviest ion species, so a plasma with $\lambda_{\mathrm{MFP}}<L$ would be collisional.

3. $\ell_{M}<\boldsymbol{L} \quad \ell_{M}$ is the magnetic diffusion lengthscale, and $L$ is the overall lengthscale of the experiment. We impose $\ell_{M}<L$ in order to ensure the magnetic field does not diffuse away during the experiment. To find $\ell_{M}$, we consider a generic diffusion equation,

$$
\frac{\partial \phi}{\partial t}=D \nabla^{2} \phi
$$

where $\phi$ is some generic diffusing quantity and $D$ is the generic diffusion constant with units $L^{2} T^{-1}$. Applying unit analysis we find

$$
\frac{\phi}{\tau}=D \frac{\phi}{\ell^{2}}
$$

where $\tau$ is the timescale of interest and $\ell$ is the diffusion length over $\tau$. This yields $\ell=\sqrt{D \tau}$.

In the case of magnetic diffusion, the diffusion constant is the magnetic diffusivity in the post-shock region, $\nu_{M}$, and the timescale of interest is $L / u$, the dynamic timescale. The magnetic diffusivity is,

$$
\nu_{M}=\frac{c^{2} \eta_{\perp}}{4 \pi}
$$

where $c$ is the speed of light, and $\eta_{\perp}$ is the transverse Spitzer resistivity. The expression for transverse Spitzer resistivity is taken from the Plasma Formulary, [18]:

$$
\eta_{\perp}=1.15 \times 10^{-14} \frac{Z \ln \Lambda}{T_{e}^{3 / 2}} \mathrm{sec},
$$

where $Z$ is the ionization, $\ln \Lambda$ is the Coulomb logarithm, and $T_{e}$ is the electron temperature in $\mathrm{eV}$. 
Altogether this yields

$$
\ell_{M}=\sqrt{\frac{\nu_{M} L}{u}}=\sqrt{\frac{c^{2} \eta_{\perp} L}{4 \pi u}}
$$

4. $\mathbf{0 . 1}<\boldsymbol{\beta}_{\text {ram }}<\mathbf{1 0} \beta_{\text {ram }}$ is the ratio of ram pressure of the jet to the magnetic pressure of the field

$$
\beta_{\mathrm{ram}}=\frac{\rho u^{2}}{B^{2} / 8 \pi},
$$

where $B$ is magnetic field strength.

A typical accreting young star system with a magnetic field of $1000 \mathrm{G}$ will have $\beta_{\text {ram }} \sim 1$. The range of acceptable $\beta_{\text {ram }}$ imposed here corresponds to magnetic field of $3000 \mathrm{G}$ (low $\beta_{\text {ram }}$ ) to $300 \mathrm{G}$ (high $\beta_{\text {ram }}$ ).

5. $\mathbf{R e}>\mathbf{1 0}^{3} \quad R e$ is the Reynolds number, the ratio of the viscous timescale to the dynamic timescale, $L u / \nu_{i}$, where $\nu_{i}$ is the ion viscosity (defined below). Imposing $R e>10^{3}$ insures that the viscous smoothing length scale, $\ell_{v}$, is smaller than all other length scales.

As before $\ell_{v}=\sqrt{D \tau}$. In this case, the diffusion constant, $D$, is the ion viscosity, $\nu_{i}$, and the timescale of interest, $\tau$ is the dynamic timescale, $L / u$,

$$
\ell_{v}=\sqrt{D \tau}=\sqrt{\frac{\nu_{i} L}{u}}=\frac{L}{\sqrt{R e}} .
$$

The ion viscosity, $\nu_{i}$, was calculated according to

$$
\nu_{i}=\frac{u_{t h, i}^{2}}{\nu_{i i}}=2 \times 10^{19} \frac{T_{i}^{5 / 2}}{n_{i} Z^{4} \sqrt{A} \ln \Lambda} \mathrm{cm}^{2} \mathrm{~s}^{-1},
$$

where $u_{t h, i}$ and $\nu_{i i}$ are the ion thermal velocity and the ion-ion collisional frequency, respectively, and expressions for both were taken from the Plasma Formulary, [18. In the expression for $\nu_{i}, T_{i}$ is the ion temperature in $\mathrm{eV}, n_{i}$ is the ion density in $\mathrm{cm}^{-3}, Z$ is the ionization, $A$ is the atomic mass, and $\ln \Lambda$ is the Coulomb logarithm.

To have a well-scaled experiment, we need these five criteria to be true at once. For every material, there is some four-dimensional volume in $T_{e}-u-\rho-B$ space where all five of these criteria are met. 
A four dimensional space is difficult to visualize, much less translate into a figure. In order to investigate the parameter space, we held $T_{e}$ and $B$ constant and considered the 2-D $u-\rho$ space, as seen in Figure 2. In each of the plots in Figure 2, the shaded area represents the region in $u-\rho$ space where the criterion is not met. Obviously, this area will shift depending on material type, temperature and magnetic field strength. We assumed that the magnetic field is $10 \mathrm{~T}$ because this is current capability; from the perspective of the dimensionless number criteria this is no downside to a higher field.

In experimenting with material and temperature, we found that every material has an ideal temperature at which the acceptable area is maximized. (For carbon this ideal temperature is around $60 \mathrm{eV}$, which is why that is shown in Figure 2.) Second, both very low-Z and very high-Z materials had smaller acceptable area than medium- $Z$ materials such as carbon. We chose to present carbon because it is a non-toxic, medium- $\mathrm{Z}$ material that lends itself readily to target fabrication.

As seen in Figure 2, the first criterion, Mach number, favors high velocity. The second criterion, collisionality, favors high density, low velocity conditions, because $\lambda_{\mathrm{MFP}} \propto 1 / n_{i}$ and $\lambda_{\mathrm{MFP}} \propto u^{4}$. The third criterion, magnetic diffusion length, favors high velocity as well because $\ell_{M} \propto 1 / u$. Magnetic diffusion length also favors higher temperatures because $\ell_{M} \propto 1 / T^{3 / 4}$. The fourth criterion, $\beta_{\text {ram }}$, favors low density, low velocity conditions because $\beta_{\text {ram }} \propto \rho$ and $\beta_{\text {ram }} \propto$ $u^{2}$. (As seen in Figure 2, it is possible for density and velocity to be too low, but the high $\beta_{\text {ram }}$ end of the range is more limiting.) The fifth and final criterion, Reynolds number, favors high velocity, high density conditions because $R e \propto u$ and $\nu_{i} \propto 1 / n_{i}$. Taken together, these criteria limit the parameter space to an area around $\rho \sim 10^{-6}-10^{-5} \mathrm{~g} \mathrm{~cm}^{-3}$ and $u \sim 100-200 \mathrm{~km} \mathrm{~s}^{-1}$ satisfies all five.

Table 1 presents the plasma parameters and calculated length scales and dimensionless numbers for the experimental plasma and the astrophysical system. 


\section{Experimental Considerations}

As seen in Figure 1, our experiment requires an incoming plasma jet (the "accreting flow") and a surface for it to run into (the "stellar surface"). This paper does not delve into the details of producing a steady carbon plasma jet with $T_{e} \sim 60 \mathrm{eV}, \rho \sim 10^{-6}-10^{-5} \mathrm{~g} \mathrm{~cm}^{-3}$, and $u \sim 100-200 \mathrm{~km} \mathrm{~s}^{-1}$. However, we note that other experimental teams have produced plasma jets made of $\mathrm{CH}$ or C, for example Gregory et al. [1] and Kuramitsu et al. [24].

Figure 3 shows the schematic of one possible experiment, based on the concept used by Gregory et al. [11, which produced jets with $\rho \sim 10^{-4} \mathrm{~g} \mathrm{~cm}^{-3}$, $T_{e} \sim 10 \mathrm{eV}$, and $u \sim 300 \mathrm{~km} \mathrm{~s}^{-1}$. In this design, lasers hit the rear side of a thin, conical target (shown in cross section), producing a collimated plasma jet that impacts against a solid block, producing shock structures for us to study. A magnetic field parallel to the direction of jet flow is imposed on the entire experiment.

Regardless of how the plasma jet is produced, the primary diagnostics for this experiment would be proton radiography, self-emission visible light imaging, and perhaps imaging Thomson scattering.

Imaging the experiment in self-emitted visible/UV light would reveal the highest temperature structures because they would glow brightest. (The opacity is too low for X-ray radiography to reveal mass density.) We would hope to see the bright shock front.

Likewise, proton radiography can be used to image magnetic field structures. Conceptually, proton radiography is similar to more traditional X-ray radiography. A source generates protons (analogous to the X-rays), which pass through the experiment and strike piece of plastic (analagous to X-ray film). We intend to use a monoenergetic-proton radiography technique described in $\mathrm{Li}$ et al. [26, 27]. A capsule of deuterium-helium-3 is imploded with high-intensity lasers, generating a burst of $14.7 \mathrm{MeV}$ and $3.6 \mathrm{MeV}$ protons. These protons pass through the experiment and strike a piece of CR-39, a clear plastic nuclear track detector, leaving a trail of broken bonds in the plastic that can be developed as 
an image when the plastic is etched.

It might be possible to use Thomson scattering to ascertain plasma parameters. The geometry of the experiment (a jet hitting a solid block) makes Thomson scattering inherently complicated. But it might be possible to bring the probe beam in the side and use imaging Thomson scattering in a transverse direction (that is, perpendicular to the jet flow direction) [40. This would tell us whether the edges of the shock (that is, the splash zone) are cooler and less dense that the central region, which would tie into the questions from Section 1 .

\section{Conclusions}

To establish the connection between a typical accreting star system and the lab, we imposed five criteria on the dimensionless numbers that underly both systems:

1. $\mathcal{M}>\mathbf{1}$ where $\mathcal{M}$ is the Mach number.

2. $\boldsymbol{\lambda}_{\mathrm{MFP}}<\boldsymbol{L}$ where $\lambda_{\mathrm{MFP}}$ is the ratio of the ion-ion mean free path.

3. $\ell_{M}<\boldsymbol{L}$ where $\ell_{M}$ is the magnetic diffusion lengthscale.

4. $\mathbf{0 . 1}<\boldsymbol{\beta}_{\text {ram }}<\mathbf{1 0}$ where $\beta_{\text {ram }}$ is the ratio of ram pressure of the jet to the magnetic pressure of the field.

5. $\boldsymbol{R e}>\mathbf{1 0}^{\mathbf{3}}$ where Re is the Reynolds number.

We find a region in parameter space where all five criteria are satisfied: a car-

bon plasma jet with $T_{e} \sim 60 \mathrm{eV}, \rho \sim 10^{-6}-10^{-5} \mathrm{~g} \mathrm{~cm}^{-3}$ and $u \sim 100-200 \mathrm{~km} \mathrm{~s}^{-1}$ with an imposed field of $B \sim 10 \mathrm{~T}$. Such an experiment would represent an average accretion stream onto a pre-main sequence star with $B \sim 700 \mathrm{G}$. (Of course, scaling accretion streams onto young stars with lower magnetic fields is easily accomplished; we can always lower the imposed experimental field.)

At $700 \mathrm{G}$, we are above maximum expected magnetic field on a Herbig Ae/Be star, usually expected to be a few hundred Gauss [48, but right at the minimum field for a $\mathrm{T}$ Tauri star, which have measured fields of several $\mathrm{kG}$ [20, 47, 49, 
19. To recreate the conditions on a $B=2000 \mathrm{G}$ star, we need $\beta_{\text {ram }}=0.3$, which requires $B \sim 30 \mathrm{~T}$, something outside the current capabilities. However, improvements in magnetic field generation could open up this experimental regime.

\section{Acknowledgements}

This work is funded by the U.S. Department of Energy, through the NNSADS and SC-OFES Joint Program in High-Energy-Density Laboratory Plasmas, grant number DE-NA0002956, and the National Laser User Facility Program, grant numbers DE-NA0002719 and R19071, and through the Laboratory for Laser Energetics, University of Rochester by the NNSA/OICF under Cooperative Agreement No. DE-NA0001944.

We thank Nuria Calvet for enlightening discussions on magnetospheric accretion and accretion shock models. 

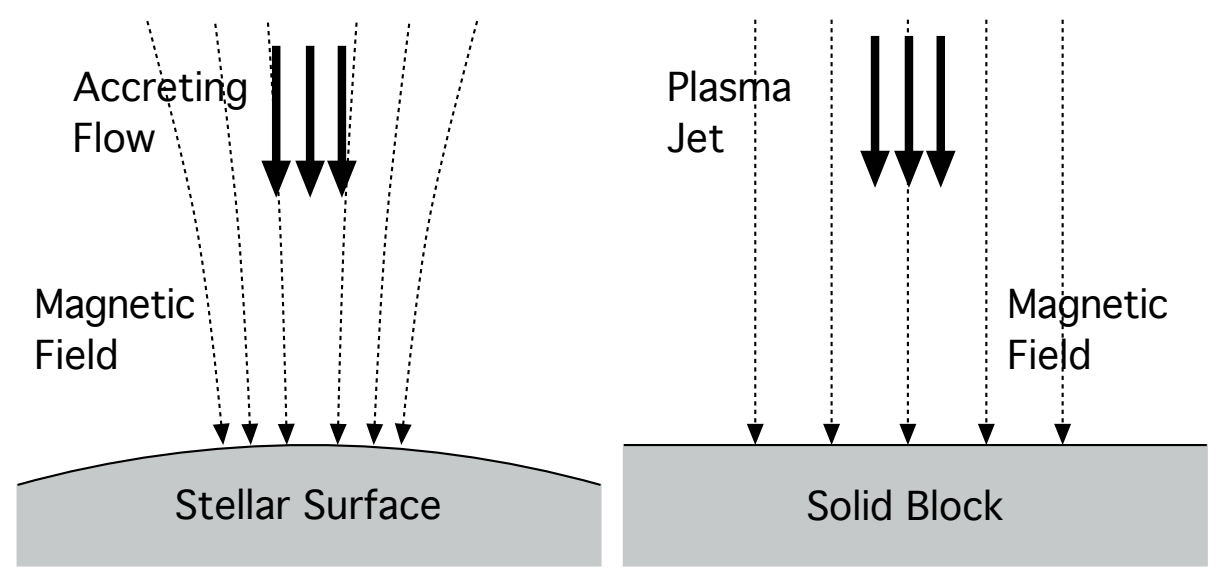

Figure 1: In the astrophysical system (left), accreting plasma falls to the stellar surface along magnetic field lines (depicted here as a magnetic dipole at the pole of the star). In the lab experiment (right), a plasma jet collides with a solid block with a parallel background magnetic field.
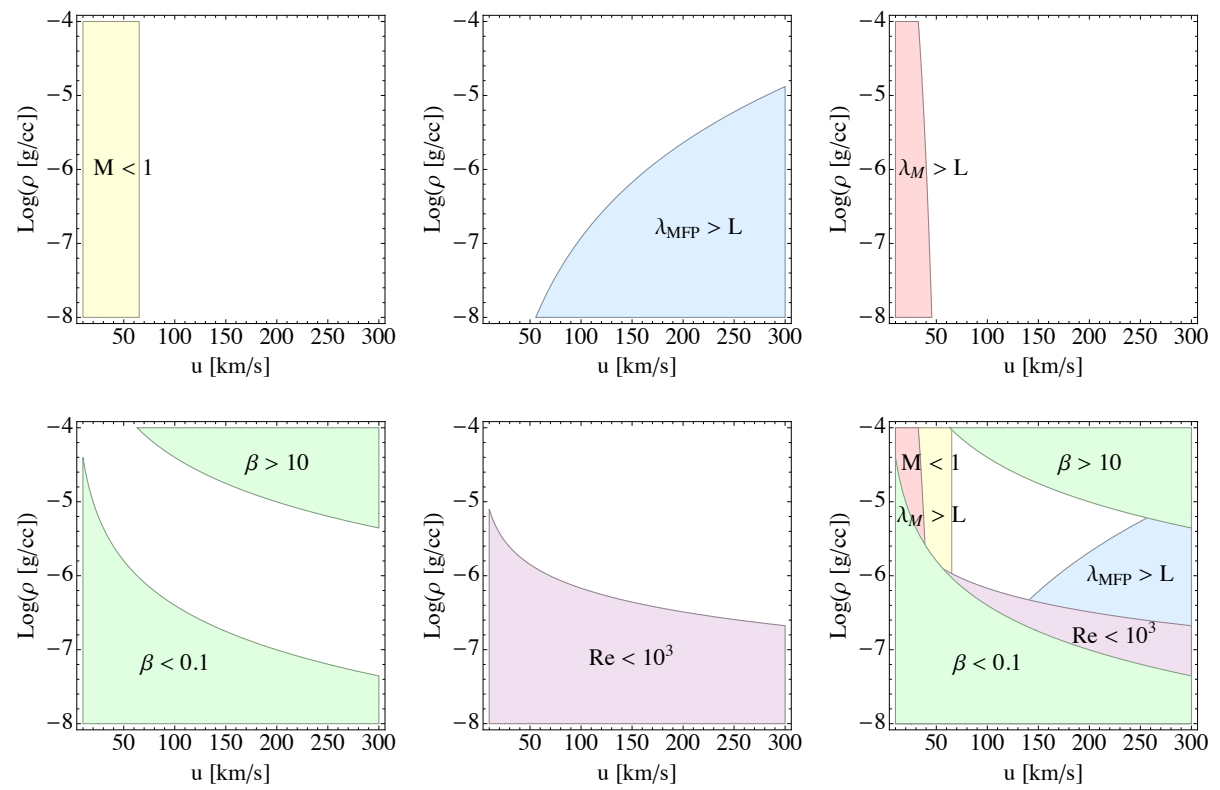

Figure 2: Criteria region plots for carbon plasma at $60 \mathrm{eV}$ with a $10 \mathrm{~T}$ magnetic field. In each plot, the colored area is the region where the conditions is not met. Top row, left to right: Mach number, collisionality, and magnetic diffusion length. Bottom row, left to right: ram plasma $\beta$, Reynolds number, and all criteria plotted together. 


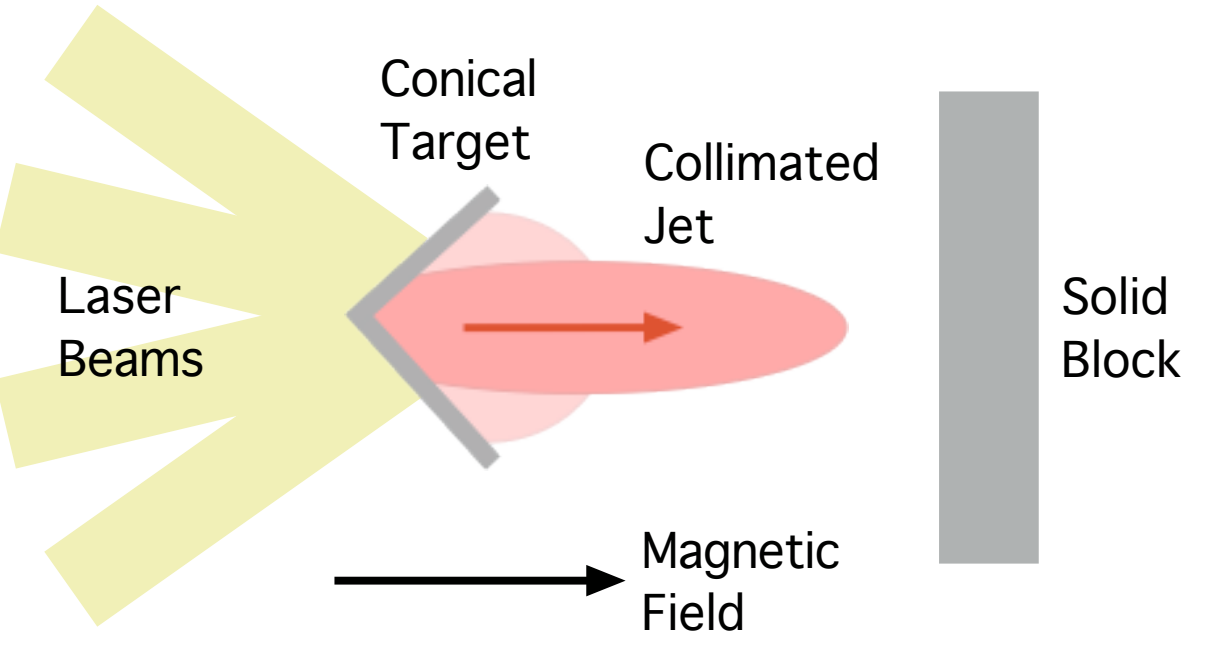

Figure 3: Schematic diagram of the experiment. Rear-irradiation launches a plasma jet toward a solid block along a parallel imposed magnetic field. 


\begin{tabular}{lccc}
\hline Parameter & Unit & Experiment & Accreting Star \\
\hline Mass density, $\rho$ & $\mathrm{g} \mathrm{cm}^{-3}$ & $5 \times 10^{-6}$ & $2 \times 10^{-11}$ \\
Average atomic number & - & 6 & 1.1 \\
Average mass number & - & 12 & 1.3 \\
Average ionization & - & 4.9 & 0.6 \\
Electron density, $n_{e}$ & $\mathrm{~cm}^{-3}$ & $10^{18}$ & $6 \times 10^{12}$ \\
Electron temperature, $T_{e}$ & $\mathrm{eV}$ & 60 & 1 \\
Velocity, $u$ & $\mathrm{~km} \mathrm{~s}^{-1}$ & 150 & 450 \\
Magnetic field strength, $B$ & $\mathrm{G}$ & $10^{5}$ & 700 \\
\hline Length scale, $L$ & $\mathrm{~cm}^{5}$ & 0.1 & $10^{9}$ \\
Ion collisional MFP, $\lambda_{\mathrm{MFP}, \mathrm{i}}$ & $\mathrm{cm}$ & 0.03 & $2 \times 10^{6}$ \\
Magnetic diffusion length, $\ell_{M}$ & $\mathrm{~cm}$ & 0.02 & 200 \\
Viscous smoothing length, $\ell_{\nu}$ & $\mathrm{cm}$ & 0.001 & 5000 \\
\hline Mach number, $\mathcal{M}$ & - & 2 & 33 \\
Collisionality, $\lambda_{\mathrm{MFP}, \mathrm{i}} / L$ & - & 0.3 & 0.002 \\
Magnetic diffusion length ratio, $\lambda_{M} / L$ & - & 0.1 & $2 \times 10^{-7}$ \\
Ram Plasma Beta, $\beta_{\mathrm{ram}}$ & - & 3 & 1 \\
Reynolds number, Re & - & $10^{5}$ & $10^{10}$ \\
Magnetic Reynolds number, $\mathrm{Re}_{m}$ & - & 20 & $10^{10}$ \\
\hline
\end{tabular}

Table 1: A side-by-side comparison of our ideal experimental jets and typical numbers in an accretion stream. The accreting star's plasma parameters are halfway in between those for T Tauri and Herbig Ae/Be stars. For the astrophysical system, velocity was assuming free-fall from the inner edge of the disk, see Calvet and Gullbring [5], Hillenbrand et al. [17, Dullemond et al. 7], Natta et al. 32. Mass density was calculated from accretion stream energy fluxes found in Calvet and Gullbring [5], Mendigutía et al. 28. Magnetic field strength was taken from Johns-Krull et al. [20], Valenti and Johns-Krull [47, Yang et al. 49], Johns-Krull 19], Wade et al. 48. 
[1] Argiroffi, C., Flaccomio, E., Bouvier, J., Donati, J.-F., Getman, K. V., Gregory, S. G., Hussain, G. A. J., Jardine, M. M., Skelly, M. B., and Walter, F. M. (2011). Variable X-ray emission from the accretion shock in the classical T Tauri star V2129 Ophiuchi. A\&A, 530:A1.

[2] Argiroffi, C., Maggio, A., and Peres, G. (2007). X-ray emission from MP Muscae: an old classical T Tauri star. A\&A, 465:L5-L8.

[3] Bouvier, J., Alencar, S. H. P., Harries, T. J., Johns-Krull, C. M., and Romanova, M. M. (2007). Magnetospheric Accretion in Classical T Tauri Stars. Protostars and Planets $V$, pages 479-494.

[4] Brickhouse, N. S., Cranmer, S. R., Dupree, A. K., Luna, G. J. M., and Wolk, S. (2010). A Deep Chandra X-Ray Spectrum of the Accreting Young Star TW Hydrae. ApJ, 710:1835-1847.

[5] Calvet, N. and Gullbring, E. (1998). The Structure and Emission of the Accretion Shock in T Tauri Stars. ApJ, 509:802-818.

[6] Drake, J. J., Braithwaite, J., Kashyap, V., Günther, H. M., and Wright, N. J. (2014). Burn Out or Fade Away? On the X-Ray and Magnetic Death of Intermediate Mass Stars. ApJ, 786:136.

[7] Dullemond, C. P., Dominik, C., and Natta, A. (2001). Passive Irradiated Circumstellar Disks with an Inner Hole. ApJ, 560:957-969.

[8] Ghosh, P. and Lamb, F. K. (1979a). Accretion by rotating magnetic neutron stars. II - Radial and vertical structure of the transition zone in disk accretion. ApJ, 232:259-276.

[9] Ghosh, P. and Lamb, F. K. (1979b). Accretion by rotating magnetic neutron stars. III - Accretion torques and period changes in pulsating X-ray sources. ApJ, 234:296-316.

[10] Grady, C. A., Hamaguchi, K., Schneider, G., Stecklum, B., Woodgate, B. E., McCleary, J. E., Williger, G. M., Sitko, M. L., Ménard, F., Henning, 
T., Brittain, S., Troutmann, M., Donehew, B., Hines, D., Wisniewski, J. P., Lynch, D. K., Russell, R. W., Rudy, R. J., Day, A. N., Shenoy, A., Wilner, D., Silverstone, M., Bouret, J.-C., Meusinger, H., Clampin, M., Kim, S., Petre, R., Sahu, M., Endres, M., and Collins, K. A. (2010). Locating the Accretion Footprint on a Herbig Ae Star: MWC 480. ApJ, 719:1565-1581.

[11] Gregory, C. D., Howe, J., Loupias, B., Myers, S., Notley, M. M., Sakawa, Y., Oya, A., Kodama, R., Koenig, M., and Woolsey, N. C. (2008). Astrophysical Jet Experiments with Colliding Laser-produced Plasmas. ApJ, 676:420-426.

[12] Grinin, V. P., Kozlova, O. V., Natta, A., Ilyin, I., Tuominen, I., Rostopchina, A. N., and Shakhovskoy, D. N. (2001). Optical spectra of five UX Orionis-type stars. A\&A, 379:482-495.

[13] Günther, H. M., Liefke, C., Schmitt, J. H. M. M., Robrade, J., and Ness, J.-U. (2006). X-ray accretion signatures in the close CTTS binary V4046 Sagittarii. A\&A, 459:L29-L32.

[14] Hartigan, P., Foster, J. M., Wilde, B. H., Coker, R. F., Rosen, P. A., Hansen, J. F., Blue, B. E., Williams, R. J. R., Carver, R., and Frank, A. (2009). Laboratory Experiments, Numerical Simulations, and Astronomical Observations of Deflected Supersonic Jets: Application to HH 110. ApJ, 705:1073-1094.

[15] Hartigan, P., Kenyon, S. J., Hartmann, L., Strom, S. E., Edwards, S., Welty, A. D., and Stauffer, J. (1991). Optical excess emission in T Tauri stars. ApJ, 382:617-635.

[16] Hartmann, L., Calvet, N., Gullbring, E., and D'Alessio, P. (1998). Accretion and the Evolution of T Tauri Disks. ApJ, 495:385.

[17] Hillenbrand, L. A., Strom, S. E., Vrba, F. J., and Keene, J. (1992). Herbig $\mathrm{Ae} / \mathrm{Be}$ stars - Intermediate-mass stars surrounded by massive circumstellar accretion disks. ApJ, 397:613-643. 
[18] Huba, J. D., States., U., and ), N. R. L. U. S. (2009). NRL plasma formulary. Naval Research Laboratory], [Washington, DC.

[19] Johns-Krull, C. M. (2007). The Magnetic Fields of Classical T Tauri Stars. ApJ, 664:975-985.

[20] Johns-Krull, C. M., Valenti, J. A., and Koresko, C. (1999). Measuring the Magnetic Field on the Classical T Tauri Star BP Tauri. ApJ, 516:900-915.

[21] Kastner, J. H., Huenemoerder, D. P., Schulz, N. S., Canizares, C. R., and Weintraub, D. A. (2002). Evidence for Accretion: High-Resolution X-Ray Spectroscopy of the Classical T Tauri Star TW Hydrae. ApJ, 567:434-440.

[22] Königl, A. (1991). Disk accretion onto magnetic T Tauri stars. ApJ, 370:L39-L43.

[23] Krauland, C. M., Drake, R. P., Kuranz, C. C., Loupias, B., Plewa, T., Huntington, C. M., Kaczala, D. N., Klein, S., Sweeney, R., Young, R. P., Falize, E., Villette, B., and Keiter, P. A. (2013). Reverse Radiative Shock Laser Experiments Relevant to Accreting Stream-Disk Impact in Interacting Binaries. ApJ, 762:L2.

[24] Kuramitsu, Y., Sakawa, Y., Waugh, J. N., Gregory, C. D., Morita, T., Dono, S., Aoki, H., Tanji, H., Loupias, B., Koenig, M., Woolsey, N., and Takabe, H. (2009). Jet Formation in Counterstreaming Collisionless Plasmas. ApJ, 707:L137-L141.

[25] Kuranz, C. C., Drake, R. P., Harding, E. C., Grosskopf, M. J., Robey, H. F., Remington, B. A., Edwards, M. J., Miles, A. R., Perry, T. S., Blue, B. E., Plewa, T., Hearn, N. C., Knauer, J. P., Arnett, D., and Leibrandt, D. R. (2009). Two-Dimensional Blast-Wave-Driven Rayleigh-Taylor Instability: Experiment and Simulation. ApJ, 696:749-759.

[26] Li, C. K., Séguin, F. H., Frenje, J. A., Rygg, J. R., Petrasso, R. D., Town, R. P. J., Amendt, P. A., Hatchett, S. P., Landen, O. L., MacKinnon, A. J., 
Patel, P. K., Smalyuk, V. A., Sangster, T. C., and Knauer, J. P. (2006).

Measuring E and B Fields in Laser-Produced Plasmas with Monoenergetic Proton Radiography. Physical Review Letters, 97(13):135003.

[27] Li, C. K., Séguin, F. H., Frenje, J. A., Rygg, J. R., Petrasso, R. D., Town, R. P. J., Landen, O. L., Knauer, J. P., and Smalyuk, V. A. (2007). Observation of Megagauss-Field Topology Changes due to Magnetic Reconnection in Laser-Produced Plasmas. Physical Review Letters, 99(5):055001.

[28] Mendigutía, I., Calvet, N., Montesinos, B., Mora, A., Muzerolle, J., Eiroa, C., Oudmaijer, R. D., and Merín, B. (2011). Accretion rates and accretion tracers of Herbig Ae/Be stars. A\&A, 535:A99.

[29] Muzerolle, J., D'Alessio, P., Calvet, N., and Hartmann, L. (2004). Magnetospheres and Disk Accretion in Herbig Ae/Be Stars. ApJ, 617:406-417.

[30] Muzerolle, J., Luhman, K. L., Briceño, C., Hartmann, L., and Calvet, N. (2005). Measuring Accretion in Young Substellar Objects: Approaching the Planetary Mass Regime. ApJ, 625:906-912.

[31] Natta, A., Grinin, V. P., and Tambovtseva, L. V. (2000). An Interesting Episode of Accretion Activity in UX Orionis. ApJ, 542:421-427.

[32] Natta, A., Prusti, T., Neri, R., Wooden, D., Grinin, V. P., and Mannings, V. (2001). A reconsideration of disk properties in Herbig Ae stars. A\&A, 371:186-197.

[33] Natta, A., Testi, L., and Randich, S. (2006). Accretion in the $\rho$-Ophiuchi pre-main sequence stars. A\&A, 452:245-252.

[34] Orlando, S., Sacco, G. G., Argiroffi, C., Reale, F., Peres, G., and Maggio, A. (2010). X-ray emitting MHD accretion shocks in classical T Tauri stars. Case for moderate to high plasma- $\beta$ values. A\&A, 510:A71.

[35] Park, H.-S., Ryutov, D. D., Ross, J. S., Kugland, N. L., Glenzer, S. H., Plechaty, C., Pollaine, S. M., Remington, B. A., Spitkovsky, A., Gargate, L., 
Gregori, G., Bell, A., Murphy, C., Sakawa, Y., Kuramitsu, Y., Morita, T., Takabe, H., Froula, D. H., Fiksel, G., Miniati, F., Koenig, M., Ravasio, A., Pelka, A., Liang, E., Woolsey, N., Kuranz, C. C., Drake, R. P., and Grosskopf, M. J. (2012). Studying astrophysical collisionless shocks with counterstreaming plasmas from high power lasers. High Energy Density Physics, 8:38-45.

[36] Remington, B. A., Drake, R. P., and Ryutov, D. D. (2006). Experimental astrophysics with high power lasers and $\mathrm{Z}$ pinches. Reviews of Modern Physics, 78:755-807.

[37] Remington, B. A., Drake, R. P., Takabe, H., and Arnett, D. (2000). A review of astrophysics experiments on intense lasers. Physics of Plasmas, $7: 1641-1652$.

[38] Robrade, J. and Schmitt, J. H. M. M. (2007). X-rays from RU Lupi: accretion and winds in classical T Tauri stars. A\&A, 473:229-238.

[39] Romanova, M. M., Ustyugova, G. V., Koldoba, A. V., and Lovelace, R. V. E. (2004). Three-dimensional Simulations of Disk Accretion to an Inclined Dipole. II. Hot Spots and Variability. ApJ, 610:920-932.

[40] Ross, J. S., Froula, D. H., Mackinnon, A. J., Sorce, C., Meezan, N., Glenzer, S. H., Armstrong, W., Bahr, R., Huff, R., and Thorp, K. (2006). Implementation of imaging Thomson scattering on the Omega Laser. Review of Scientific Instruments, 77(10):100000.

[41] Ryutov, D., Drake, R. P., Kane, J., Liang, E., Remington, B. A., and WoodVasey, W. M. (1999). Similarity Criteria for the Laboratory Simulation of Supernova Hydrodynamics. ApJ, 518:821-832.

[42] Schmitt, J. H. M. M., Robrade, J., Ness, J.-U., Favata, F., and Stelzer, B. (2005). X-rays from accretion shocks in T Tauri stars: The case of BP Tau. A\&A, 432:L35-L38. 
[43] Stelzer, B. and Schmitt, J. H. M. M. (2004). X-ray emission from a metal depleted accretion shock onto the classical T Tauri star TW Hya. A\&A, 418:687-697.

[44] Swartz, D. A., Drake, J. J., Elsner, R. F., Ghosh, K. K., Grady, C. A., Wassell, E., Woodgate, B. E., and Kimble, R. A. (2005). The Herbig Ae Star HD 163296 in X-Rays. ApJ, 628:811-816.

[45] Testa, P., Huenemoerder, D. P., Schulz, N. S., and Ishibashi, K. (2008). XRay Emission from Young Stellar Objects in the $\epsilon$ Chamaeleontis Group: The Herbig Ae Star HD 104237 and Associated Low-Mass Stars. ApJ, 687:579597.

[46] Valenti, J. A., Basri, G., and Johns, C. M. (1993). T Tauri stars in blue. AJ, 106:2024-2050.

[47] Valenti, J. A. and Johns-Krull, C. M. (2004). Observations of Magnetic Fields on T Tauri Stars. Ap\&SS, 292:619-629.

[48] Wade, G. A., Bagnulo, S., Drouin, D., Landstreet, J. D., and Monin, D. (2007). A search for strong, ordered magnetic fields in Herbig Ae/Be stars. MNRAS, 376:1145-1161.

[49] Yang, H., Johns-Krull, C. M., and Valenti, J. A. (2005). Measuring the Magnetic Field of the Classical T Tauri Star TW Hydrae. ApJ, 635:466-475. 